\title{
Thermomechanical Response of Actively Cooled Metal Foam Sandwich Panels for Thermal Protection Systems
}

\author{
Joseph F. Rakow * and Anthony M. Waas ${ }^{\dagger}$ \\ Composite Structures Laboratory, \\ Department of Aerospace Engineering, \\ University of Michigan, Ann Arbor, MI 48109-2140
}

\begin{abstract}
With a favorable strength-to-weight ratio and high specific internal surface area, sandwich panels with metal foam cores are proposed for actively cooled load-bearing components in aerospace thermal protection systems. The design of such a system requires predictive tools developed and verified on relevant hardware. As yet, existing experimental investigations that address the thermomechanical response of actively cooled sandwich panels, regardless of core construction, are very limited in number and focus primarily on fatigue behavior. In addition, the majority of work related to the thermomechanical response of sandwich panels, in general, and even that related to monolithic panels is theoretical and computational in nature, with little or no experimental validation. The present work presents experimental data for the thermomechanical response of actively cooled metal foam sandwich panels (MFSP's). First, inconel foam sandwich panels are subjected, via experimental methods, to through-the-thickness thermal gradients, defined by fixed temperature conditions on opposite face sheets. With clamped boundary conditions, provided through a novel test fixture designed to minimize the common conflict of thermal and mechanical boundary conditions in experimental apparatus, the MFSP's exhibit out-ofplane bending deformation. Control of thermomechanical deformation within the panel is then demonstrated through active cooling, achieved by pumping compressed air through the metal foam core. Subsequently, the experimental measurements are used to develop a sequentially coupled thermomechanical finite element model. Temperature-dependent material data is included in the model for the elastic-plastic regime encountered experimentally. Central to the numerical characterization of MFSP's is an understanding of the response of metal foam under shear loading. The material model for the foam is taken from a series of experimental measurements of the shear response of metal foam, providing density-dependent relationships for material stiffness and strength. The numerical model provides a strain-temperature history for MFSP's under through-the-thickness thermal gradients and the results are shown to agree very well with the experimental measurements.
\end{abstract}

\footnotetext{
${ }^{*}$ Graduate Research Assistant, Copyright (C)2005 Joseph F. Rakow and Anthony M. Waas, Published by the AIAA with permission

$\dagger$ Professor of Aerospace Engineering, University of Michigan, Associate Fellow, AIAA, corresponding author
} 


\section{Introduction}

\section{A. Current Thermal Protection Systems}

Thermal protection during high speed flight is one of the most pressing issues in the advancement of hypersonic vehicles. Since the early 1950's, with the development of the $X-2$ and $X-15$ vehicles, designed to capitalize on the supersonic accomplishment of the $X-1$, until recent years, marked by the disintegration of the space shuttle Columbia during hypersonic re-entry, the technology to propel vehicles has been more mature and more successful than the technology to protect vehicles from the resulting, tremendous aerodynamic heat loads. This limitation has manifested and dominated a variety of aspects of hypersonic vehicle operation - from flight planning to vehicle maintenance to flight performance envelopes - examples of each are as follows. Flight planning: the ceramic thermal protection system (TPS) on the space shuttle cannot fly in wet weather because of its hygroscopic surface. Vehicle maintenance: the TPS on the space shuttle, as a whole, dominates the maintenance requirements of the vehicle, demanding $32,000^{1}$ of the 50,000 man-hours of inspection and repair required by the vehicle between flights. Performance envelopes: on its record-setting flight above Mach 6, components of the $X-15$ were virtually destroyed when shock-shock interactions pierced holes in the inconel airframe, a heat sink TPS. As illustrated by these examples the current state-of-the-art in TPS technology cannot efficiently manage the demanding requirements of hypersonic vehicle operation.

One concept for advanced structural thermal protection systems, and the one that promises the highest magnitude of thermal load management, ${ }^{2}$ is an actively cooled thermal protection system, in which load bearing structural members are integrated with coolant passages. Unlike the dual-component ablative TPS onboard the manned capsules of the Mercury, Gemini, and Apollo aircraft and unlike the dual-component insulated system employed on the space shuttle, an ideal structural thermal protection system is a singlecomponent multifunctional structure that bears both mechanical and thermal loads.

To this point in time, however, true integration and multifunctionality have not been achieved. An example of a first-generation actively cooled structural panel is shown in Figure $1 .{ }^{3}$ This panel is a standard, honeycomb-cored sandwich panel that has been modified with a series of coolant tubes on the inside of the external face sheet. The problems associated with this type of construction are readily apparent:

1) The coolant tubes interrupt the path for shear load transfer between the face sheets and the honeycomb core.

2) Machining of the honeycomb and the lay-up of the panel to accommodate these tubes create significant manufacturing difficulties.

3) The discreet nature of the coolant passages causes severe thermal stress gradients throughout the panel.

4) Blockage in discretized coolant passages leads to local hot spots of reduced strength in the load-bearing structure.

For success, such problems must be overcome in next-generation actively cooled TPS concepts.

\section{B. Improvements Offered by Metal Foam for Thermal Protection Systems}

Metal foam sandwich panels (MFSP's) promise a truly multifunctional structure that is well-suited for the management of both thermal and mechanical loads. An actively cooled MFSP is a sandwich panel with an open-cell metal foam core integrally bonded (i.e.- brazed) to metal face sheets. While the panel bears airframe flight loads, aerodynamic heat on the panel's outer surface is conducted through the outer face sheet and, with the assistance of the conductive foam, into coolant passing through the core of the panel. A schematic of this arrangement is shown in Figure 2.

Metal foam, which bears mechanical shear loads within the MFSP while conductively distributing heat from the outer surface of the panel into and throughout the cross-sectional flow of the coolant, is a critical component of the multifunctional panel. In its open cell form, metal foam has a sub-structure similar to 
foam found in seat cushions and packing materials, but the sub-structure is made of a metallic alloy (i.e.aluminum, titanium, inconel, copper). Figure 3 shows an open cell metal foam in detail. In previous studies, the present authors have demonstrated and characterized the response of metal foams under shear loading through experimentation, ${ }^{4}$ numerical simulation, ${ }^{5}$ and a micromechanics-based analysis. ${ }^{6}$

Metal foam is central to the improvements offered by MFSP's over previous actively cooled panel concepts:

1) The foam and face sheets provide a monolithic, single-material structure with no inherent intrastructural mismatch of coefficients of thermal expansion.

2) Through-the-thickness shear response and heat transfer can be controlled by controlling the foam density.

3) The metal foam core provides a network of non-discreet coolant passages, eliminating the severity of local coolant blockage.

4) With high internal surface area per unit volume $\left(\sim 1800 \frac{\mathrm{m}^{2}}{\mathrm{~m}^{3}}\right)$, metal foam facilitates extremely high structure-coolant interaction per unit volume.

5) In addition to conducting heat, the foam core improves the heat transfer capability of the system by creating turbulence in the coolant, even at low Reynolds numbers. The departure from laminar flow introduces a non-zero eddy coefficient, $\epsilon_{H}$, into the boundary layer equations for steady, incompressible flow with constant properties, which increases the rate of heat flux, $\overrightarrow{\mathbf{Q}}$, to the coolant,

$$
\overrightarrow{\mathbf{Q}}=-\rho c_{p}\left(\alpha+\epsilon_{H}\right) \frac{\partial \bar{T}}{\partial z} .
$$

In (1), $\rho, c_{p}$, and $\alpha$ are the density, specific heat at constant pressure, and thermal diffusivity of the coolant, respectively, and $\frac{\partial \bar{T}}{\partial z}$ is the time-averaged thermal gradient field perpendicular to the flow. ${ }^{7}$

\section{Thermomechanical Response of Actively Cooled MFSP's}

The present work demonstrates and analyzes a fully functioning prototype actively cooled MFSP. The core of this work is a thermostructural experiment and a finite element analysis of an MFSP with inconel face sheets and an inconel foam core subjected to active cooling with compressed air. Inconel, chosen for its proven structural integrity under high heat flux and high structural temperatures, has served as a primary material for structural components on previous hypersonic aircraft such as the $X-15$. In the experiments and in the numerical model of the present work, the specimen is subjected to a through-the-thickness thermal gradient under mechanical constraints and the response of the panel is measured. Subsequently, control of this response through active cooling is demonstrated. The response of MFSP's to through-the-thickness thermal gradients is critical for hypersonic vehicle operation as external structural members are subjected to severe through-the-thickness thermal gradients due to aerodynamic frictional heating on the outside of the craft and the necessarily cooler conditions on the interior of the craft. Such thermal loading causes deformation on both the heated and unheated sides of structural components.

In the following section, inconel MFSP's are subjected to through-the-thickness thermal gradients, constrained deformation, and active cooling with compressed air. The thermomechanical response of the cooled and uncooled panels is captured with strain gages and distributed thermocouples. The results demonstrate an ability to control and, under certain conditions, completely eliminate the thermostructural deformation of the MFSP's through active cooling.

In conducting this full-scale experiment - an MFSP with a through-the-thickness thermal gradient, constrained deformation, and active cooling - it is necessary to determine appropriate flow conditions for active cooling. These efforts are discussed in the appendix of the present papera. Within that work, two

\footnotetext{
${ }^{a}$ The work contained in the appendix was carried out with the cooperation of three undergraduate students under the direction of the present authors. The authors are grateful to Yanyu Luo (2003), Ken Salas (2004), and Kristen Montz (2004) for conducting the experimental work.
} 
parameters are studied with respect to the thermal performance of the actively cooled MFSP: 1) the effect of foam core density, 2) the effect of pressure drop and, therefore, flow rate. In each of the studies, MFSP's are subjected to a through-the-thickness thermal gradient and are cooled with compressed air. The results provide an indication of the airflow rates required for substantial cooling of the inconel MFSP's analyzed in the present work.

In section III, a finite element model is developed to analyze the experimental actively cooled inconel MFSP under thermal-structural loading. The model is three-dimensional with temperature-dependent elastoplasticity and shows excellent agreement with the experimental results.

\section{Experimental Investigation}

\section{A. Procedure}

The experimental test article is shown in Figure 4. The MFSP is a square panel, measuring $292 \times 292 \mathrm{~mm}$ $(11.5 \times 11.5 \mathrm{in})$, comprised of two inconel 625 face sheets, $1 \mathrm{~mm}(0.04 \mathrm{in})$ thick, with an inconel 625 foam core that has a thickness of $6.4 \mathrm{~mm}(0.25 \mathrm{in})$, a relative density of $\rho=8 \%$, and 20 pores per inch. Also shown in Figure 4 is the cooling manifold that will be used to deliver the cooling air to the core of the MFSP. Figure 5 shows the test article and the cooling manifold mounted in a clamping frame made of $6 \mathrm{Al} 4 \mathrm{~V}$ titanium. Titanium is necessary for the frame because the frame must have a lower coefficient of thermal expansion than the test article in order to ensure contact between the frame and the MFSP throughout the experiment. The frame has two identical pieces that are clamped together to provide a clamped boundary condition along the border of the MFSP. This configuration creates a central square test section measuring $216 \times 216 \mathrm{~mm}$ $(8.5 \times 8.5 \mathrm{in})$. In addition to providing clamped boundary conditions around the perimeter of the test article, the frame also provides a leak-proof seal for the coolant flowing through the MFSP. Additional benefits of this clamping frame, particularly with respect to experimental thermomechanical boundary conditions, are discussed in detail by the present authors in previous publications. ${ }^{8,9}$

The frame-panel assembly is subjected to thermal loading in a dual-platen heat press for which the temperature of each platen is programmable and may be controlled independently. A diagram of the panelframe assembly in the heat press is shown in Figure 6 with an image of the actual heat press shown in Figure 7. The top platen (labeled "Hot Platen" in Figure 6 and Figure 7) provides heating to the test article, simulating the external heating that would be encountered in a high-speed air vehicle. The temperature of the top platen is set to one of several pre-determined temperatures, as addressed below. The bottom platen (labeled "Cool Platen") is uncontrolled in temperature, but provides thermal inertia for the bottom surface of the panel. These conditions on the bottom surface of the test article simulate the conditions present on the interior of a high-speed air vehicle. For the remainder of this discussion, the side of the panel-frame assembly subjected to direct heating from the hot platen will be called the exterior, as in the exterior of the vehicle. The side of the panel-frame assembly not subjected to direct heating (i.e.- the side in contact with the cool platen) will be called the interior, as in the interior of the vehicle.

A sample thermal loading profile used in the experiments is shown in Figure 8. At point A, the MFSP, the clamping frame, and the heat press are each at room temperature. Between point $\mathrm{A}$ and point $\mathrm{B}$, the hot platen of the heat press is heated until it reaches a predefined temperature. Meanwhile, the panel-frame assembly is intentionally insulated from the hot platen so that it remains at room temperature. Once the target temperature is reached on the hot platen, the exterior side of the uncooled MFSP, as well as the exterior side of the frame, are put in contact with the hot platen of the heat press, while the interior side of the MFSP and frame are in contact with the cool platen. This occurs at point B. Between point B and point $\mathrm{C}$, a dynamic thermal gradient develops through the thickness of the MFSP and the temperature of the panel increases until thermal equilibrium is established, which occurs at point $\mathrm{C}$. The data collected between point $\mathrm{B}$ and point $\mathrm{C}$ represent the response of an uncooled MFSP to through-the-thickness thermal gradients. Note that the equilibrium temperature of the exterior side of the MFSP and of the exterior side of the frame are below the temperature of the hot platen. This is attributed to the fact that the experimental setup is not 
insulated - heat is allowed to escape to the atmosphere. At point $C$, the cooling is activated and remains activated, causing the temperature of the assembly to drop, reaching a new thermal equilibrium (point D) associated with the actively cooled MFSP. At point $\mathrm{D}$, the experiment is terminated. The thermomechanical response of the MFSP with active cooling, compared to that without cooling, demonstrates the effectiveness of the actively cooled MFSP in managing thermostructural deformation.

Three experiments are conducted, each at a different fixed temperature for the hot platen: $260^{\circ} \mathrm{C}, 330^{\circ} \mathrm{C}$, $540^{\circ} \mathrm{C}$. The hot platen temperatures are chosen based on the limitations of the instrumentation (i.e.- strain gages) and the heat press. The maximum service temperature of the instrumentation is near $330^{\circ} C$, so the first two temperatures explore the range of measurable structural deformations. The third temperature $\left(540^{\circ} \mathrm{C}\right)$ is the upper temperature limit of the heat press. Under the condition of $540^{\circ} \mathrm{C}$, the strain gages on the exterior side of the test article will overheat and be destroyed. However, the gages on the interior side of the panel will not overheat before the experiment is complete. Therefore, valuable information may be captured on the interior side of the test article at this elevated temperature.

As previously discussed, the test article is fitted with a manifold to allow for compressed air to be passed through the core of the MFSP. The compressed air is maintained at room temperature under $6.9 \mathrm{MPa}(1000 \mathrm{psi})$. The flow rate and the gage pressure of the air passed through the panel are regulated to be constant for each experiment - $30 S C F M^{\mathrm{b}}$ and $69 \mathrm{kPa}(10 \mathrm{psi})$. Once through the panel, the air is simply out-gassed to the laboratory surroundings.

The panel-frame assembly is instrumented with a series of back-to-back strain gages and distributed thermocouples in the locations depicted in Figure 9. The strain gages provide in-plane deformation measurements and the back-to-back pairs provide an indication of out-of-plane deformation. The distributed thermocouples provide temperature histories for the MFSP and for the frame. In addition, this network of temperature measurements will serve as the thermal loading input for the finite element analysis of section III.

\section{B. Results and Discussion}

Figure 10 and Figure 11 show the response of the inconel MFSP when subjected to a hot platen temperature of $260^{\circ} \mathrm{C}$ and $330^{\circ} \mathrm{C}$, respectively, and then actively cooled. The initial plateau (point A to point $B$ ) represents the time before the panel is exposed to the hot platen. Once exposed, at point B, a strain response rapidly develops on both the hot and cool sides of the panel. This strain is a combination of tensile thermal strain and compressive mechanical strain due to the titanium frame. As is clear from the plots, the interior and exterior sides of the MFSP have qualitatively similar responses at different magnitudes.

As the time of exposure to thermal loading increases, thermal equilibrium is reached throughout the panel, which translates to mechanical equilibrium demonstrated by the peak plateau response at point C. The difference between the strain levels on the interior and exterior sides of the panel, throughout the response, indicates a combination of thermal bending and of thermal shearing of the panel. The magnitude of this value increases with time until the steady state is reached. This initial response curve indicates the response of the inconel MFSP without any active cooling.

After the plateau response has been reached, the cooling is activated at point $\mathrm{C}$ and the strain levels on both the interior and exterior sides of the panel drop dramatically. In fact, point D in Figure 10 and Figure 11 indicates that, by way of active cooling, the strain level on the interior side of the panel is brought back to its initial, unheated configuration even though the specimen is still exposed to the fixed temperature of the hot platen. This is an important result. The data in Figure 10 and Figure 11 demonstrate that active cooling can effectively manage the structural temperature and deformation on the interior side of the panel such that the effects of external heating are no longer present.

As indicated above, the maximum operating temperature of the strain transducers is approximately $330^{\circ} \mathrm{C}$. When the exterior side of the panel is subjected to $540^{\circ} \mathrm{C}$, as in the third experiment, the sensors on the top side of the panel become destroyed and provide no useful data. However, the gages on the interior

\footnotetext{
${ }^{\mathrm{b}} \mathrm{SCFM}$ - Standard Cubic Feet per Minute
} 
side of the panel never reach $330^{\circ} \mathrm{C}$. It is possible, then, to obtain response measurements for the cool side of the panel when the hot side is subjected to very high temperatures. A maximum temperature of $540^{\circ} \mathrm{C}$ is chosen because this is the maximum temperature of the heat press used in these experiments.

Figure 12 shows the response of the inconel MFSP when the exterior side of the panel is subjected to a temperature of $540^{\circ} \mathrm{C}$. As in the first two experiments, the initial strain response of the panel (point $\mathrm{B}$ to point C) increases on each side of the MFSP without active cooling, until equilibrium is established (point C). Within this response, Figure 12 indicates the point at which the strain gages on the exterior side of the panel are destroyed. Once the unheated MFSP reaches thermal equilibrium at point $\mathrm{C}$, the cooling is activated and the response of the panel changes dramatically, eventually reaching a new equilibrium configuration at point D. Again, even with the temperature of $540^{\circ} \mathrm{C}$ on the exterior side of the panel, the structural response of the cool side of the panel can be controlled through active cooling such that the effects of external heating are no longer present.

These results are extremely promising. Certainly, at some external temperature greater than $540^{\circ} \mathrm{C}$, the temperature of the interior side (i.e.- the payload side) of the actively cooled MFSP will rise above room temperature and that side of the panel will exhibit an elevated thermomechanical response. In this case, the flow rate of compressed air could also be increased. It is of interest to extend the experiments in the present work to greater external temperatures, akin to those experienced in extreme hypersonic environments. Such work is necessary to qualify actively cooled MFSP's for load bearing thermal protection systems.

One interesting aspect of the response curves shown in Figure 10, Figure 11, and Figure 12 is that the structural response is essentially actuated by the active cooling. To demonstrate the controllable nature of this system, the results of a novel series of experiments are presented. In these experiments, the uncooled panel is heated and the rapid initial response of the MFSP is measured, identical to the first set of experiments. With the temperature of the hot platen constant throughout the experiment and before reaching thermal equilibrium, the cooling is activated and the magnitude of the strain response decreases, as before. The cooling air is cycled on and off, the strain levels fluctuate accordingly, and actuation of the structural deformation, through active cooling, is demonstrated. These response curves are presented in Figure 13 and Figure 14 for exposure to temperatures of $260^{\circ} \mathrm{C}$ and $330^{\circ} \mathrm{C}$, respectively.

\section{Numerical Investigation}

In this section, a finite element model of the experimental panel-frame assembly will be developed and analyzed. Once validated with respect to the existing experimental data, the model provides a forum for investigating critical design parameters such as materials and geometry, including foam density. In addition, and more immediately relevant, the model allows for further investigation into the dominant deformation mechanisms that produced the experimental results observed in Section II.

The primary interest of the numerical effort is to model the mechanical response of the actively cooled inconel MFSP's. The experimental temperature histories of the MFSP and of the clamping frame (Figure 8), as measured by the distributed thermocouples in the experiments described in section II, are entered into the finite element model to serve as the thermal loading. These temperature histories are captured from multiple points on the MFSP and on the frame (see Figure 9). The measured experimental temperature data is directly entered into the finite element input file in tabular form. The strain response of the MFSP to this thermal loading, as predicted by the analysis, is then compared to the response measured experimentally and presented in Figure 10, Figure 11, and Figure 12.

\section{A. Geometry, Mesh, Boundary Conditions, and Loading}

The experimental load frame has two planes of symmetry with respect to geometry, boundary conditions, loading, and modes of deformation throughout the experiment. This allows for the finite element model to consist of a quarter of the panel-frame assembly, as shown in Figure 15. Both the MFSP and the frame are modeled entirely with three-dimensional linear brick elements (C3D8), using the commercial finite element 
code, ABAQUS. The solution is obtained through a sequentially coupled thermal-stress solution algorithm, in which the heat transfer problem is solved first, providing nodal temperatures for each node in the model. These temperatures are then used for thermal loading in the solution of the mechanical problem. The model has approximately 36,000 elements and 128,000 degrees of freedom.

The MFSP is modeled as a solid, partitioned into three regions, two for the face sheets and one for the core. The core and the face sheets of the MFSP each have three elements through the thickness. The foam core is modeled as a homogeneous isotropic solid that has effective temperature-dependent properties based on experimental measurements conducted by the present authors. Detailed discussion of this and other material issues are addressed in the following section.

Boundary conditions for all nodes on each plane of symmetry are such that

$$
u_{n}=0,
$$

in which $u_{n}$ is the component of displacement normal to the plane of symmetry. The connection between the MFSP and the frame is modeled with a series of tie contacts, for which the frame nodes are the master nodes and the MFSP nodes are the slave nodes. These tie contacts represent perfect bonding, such that,

$$
\vec{u}_{\text {panel }}=\vec{u}_{\text {frame }}
$$

along the entire interface between the panel and the frame. In addition, with $z$ taken to be the direction perpendicular to the surface of the MFSP,

$$
\vec{u}_{z}=0
$$

along the interface between the clamping frame and the heat press platens.

\section{B. Material Properties}

The face sheets are made of inconel 625. Temperature-dependent stress-strain curves for inconel 625 are available from material databases ${ }^{10}$ and are shown in Figure 16. It is clear from this figure that the linear elastic response of inconel 625 does not change significantly within the temperature range of the experiments involved in the present work $\left(24^{\circ} \mathrm{C}-330^{\circ} \mathrm{C}\right)$. In the model, then, the elastic properties are assumed to be independent of temperature over the temperature range to be modeled. Likewise, plastic deformation is not allowed in the face sheets because the measured experimental strains did not detect the presence of plastic deformation. It is further confirmed, through this finite element model, that the stress levels in the face sheets do not approach the material yield point. Experimental measurements have been obtained by the present author for the coefficient of thermal expansion (CTE) of the inconel face sheets and of the titanium frame over the range of temperatures encountered in the present experiment. It is found that these values are $\alpha_{\text {inconel }}=12.8 \mu \epsilon /{ }^{\circ} \mathrm{C}$ and $\alpha_{T i}=9.0 \mu \epsilon /{ }^{\circ} \mathrm{C}$ and are constant over the temperature range encountered in the present experiments. This information is used for the CTE of inconel in the simulation.

Like the face sheets, the foam core is made from inconel 625 . The core has 20 pores per inch and a relative density of $\rho_{f}=8 \%$. Elastic properties for the inconel foam in the model are scaled from elastic properties that have been determined experimentally by the present authors for aluminum foam of like construction. ${ }^{4,8,9}$ Appropriate scaling relations take on the following form:

$$
\frac{E_{\text {inconel }}^{\text {foam }}}{E_{\text {aluminum }}^{\text {foam }}}=\frac{E_{\text {inconel }}^{\text {solid }}}{E_{\text {aluminum }}^{\text {solid }}}
$$

The inconel foam core remains completely elastic throughout the thermal loading history in the experiment and in the model. In addition, the inconel foam core is assumed to have the same CTE as the inconel face sheets, which is a commonly accepted assumption regarding metal foam. ${ }^{11}$ 


\section{Results and Discussion}

Figure 17 and Figure 18 show the experimental data and the results of the finite element simulation. The plots indicate very good agreement in all phases of the experiment.

The favorable nature of the agreement between experiment and simulation shown in Figure 17 and Figure 18 may be attributed, in part, to the fact that, while a large portion of the strain magnitude is due to thermal expansion, the simulated thermal loading in the MFSP and in the frame is defined by the temperature history measured experimentally. The strain due to thermal expansion is governed by the CTE of the material and the temperature change, both of which have been included in the model, a prioi, based on experimental measurements. Nonetheless, the simulation clearly captures the thermomechanical deformation present in the experiment.

With agreement established between the finite element model and the experiments, the results of Figure 12 , in which the maximum operating temperature of the strain gages is exceeded, may now be revisited and completed. The reader may recall that the results of Figure 12 were obtained under experimental thermal conditions that destroyed the strain gages on the side of the MFSP directly exposed to the hot platen. The experiment was pursued, despite this fact, because valuable data could still be obtained on the interior (i.e.cooler) side of the MFSP. Now, with the finite element model, deformation data for the exterior side of the MFSP may be obtained to complement the data obtained experimentally on the interior side of the panel. These results are shown in Figure 19.

\section{Conclusions}

The present work demonstrates and quantifies the effectiveness of actively cooled MFSP's in providing thermal protection while bearing mechanical load. In the present work, inconel MFSP's are subjected to through-the-thickness temperature gradients in a manner that simulates conditions to be expected in primary fuselage panels of high-speed vehicles. In this sense, one side of the panel is considered the exterior of the vehicle and is subjected to a predetermined temperature. The other side of the panel is considered the interior of the vehicle - its temperature is not moderated by a heat source. This investigation demonstrates that active cooling can eliminate elevated temperatures and thermomechanical deformation on the unheated side of an MFSP (i.e.- the interior of the fuselage panel) for temperature exposure as high as $540^{\circ} \mathrm{C}$ on the exterior side of the panel. In addition, the thermomechanical deformation on the exterior side of the panel is greatly reduced via active cooling. It is reasonable to conclude that, in test facilities capable of applying higher temperatures and greater coolant flow rates, such favorable performance could be demonstrated under more extreme thermal environments, such as those that approach the thermal limits of the metallic structural material ${ }^{\mathrm{c}}$. In addition to demonstrating thermal protection, these results reveal an ability to actuate, through active cooling, the thermomechanical deformation of MFSP's.

The experimental results are complemented with a finite element analysis. The results of the simulation are in very good agreement with those measured experimentally for all phases of the thermomechanical response, with and without active cooling. Upon establishing agreement for relatively low temperature environments, the model is shown to be useful for investigating the thermomechanical response of the MFSP's under thermal environments that exceed the operating limits of the experimental apparatus.

\section{Acknowledgements}

This work was supported by the Space Vehicle Technology Institute under grant NCC3-989, jointly funded by NASA and DoD within the NASA Constellation University Institutes Project, with Claudia Meyer as the project manager.

\footnotetext{
${ }^{\mathrm{c}}$ Inconel, with a melting point of $1300^{\circ} \mathrm{C}$, performed successfully at temperatures of $700^{\circ} \mathrm{C}$ on the $X-15$.
} 


\section{References}

${ }^{1}$ S.J. Scotti. Thermal protection systems for reusable launch vehicles. Symposium on Structures for Hypersonic Vehicles, ASME International Congress, November 2003.

${ }^{2}$ H.N. Kelly and M.L. Blosser. Active cooling from the sixties to NASP. Current Technology for Thermal Protection Systems, NASA CP-3157:189-249, 1992.

${ }^{3}$ D.A. Ellis, L.L. Pagel, and D.M. Schaeffer. Design and fabrication of a radiative actively cooled honeycomb sandwich structural panel for hypersonic aircraft. Technical Report CR-2957, NASA, 1978. 2004.

${ }^{4}$ J.F. Rakow and A.M. Waas. Size effects and the shear response of aluminum foam. Mechanics of Materials, 37(1):297-310,

${ }^{5}$ J.F. Rakow and A.M. Waas. Size effects in metal foam cores for sandwich structures. AIAA Journal, 42(7):1331-1337, July 2004.

${ }^{6}$ J.F. Rakow and A.M. Waas. The effective isotropic moduli of random fibrous composites, platelet composites, and foamed solids. Mechanics of Advanced Materials and Structures, 11(2):151-173, 2004.

${ }^{7}$ F.P. Incropera and D.P. DeWitt. Fundamentals of Heat and Mass Transfer. John Wiley and Sons, New York, NY, fifth edition, 2002.

${ }^{8}$ J.F. Rakow and A.M. Waas. Thermal buckling of metal foam sandwich panels for actively cooled thermal protection systems. Accepted for publication in Journal of Spacecraft and Rockets. Originally presented at AIAA 45th Structures, Structural Dynamics, and Materials Conference, April 2004, Palm Springs, CA, Paper Number AIAA-2004-1710, 2004.

${ }^{9}$ J.F. Rakow. Thermomechanical Response of Metal Foam Sandwich Panels for Structural Thermal Protection Systems in Hypersonic Vehicles. University of Michigan, 2005. Doctoral Dissertation.

${ }^{10} \mathrm{C}$. Moosbrugger, editor. Atlas of Stress-Strain Curves. ASM International, 2002.

${ }^{11}$ M.F. Ashby, A. Evans, N.A. Fleck, L.J.Gibson, J.W. Hutchinson, and H.N.G. Wadley. Metal Foam: A Design Guide. Butterworth-Heinemann, Boston, MA, 2000. 


\section{Appendix}

Before conducting a full thermostructural response experiment for the actively cooled MFSP, it is valuable to acquire a qualitative understanding of the thermal performance of MFSP's so that system parameters may be chosen appropriately for the experimental specimen and procedure. The work addressed below is an investigation into the effects on the thermal performance of MFSP's of varying two primary system parameters - metal foam density and coolant flow rate. This work has been conducted in cooperation with three undergraduate students, Yanyu Luo (2003), Ken Salas (2004), and Kristen Montz (2004).

\section{A. Experimental Procedure}

Five MFSP's are used in the study, spanning a range of foam densities of $\rho_{f}=5.5,8.0,9.0,10.2,12.2 \%$. The panels have the same cross-sectional geometry as the inconel MFSP's studied throughout the present paper $-6.4 \mathrm{~mm}$ core thickness with $1 \mathrm{~mm}$ face sheet thickness. The materials are 6061-T6 aluminum for the face sheets and 6101-T6 aluminum for the core. The panels measure $203.3 \mathrm{~mm}$ in length and $50.8 \mathrm{~mm}$ in width.

The experimental articles are tested in the set-up depicted in Figure 20. One side of the panel is heated by a $500 \mathrm{~W}$ heat lamp and all other sides of the panel are free convection boundaries. The panel is fitted with a manifold so that compressed air may be passed through the foam core and freely out-gassed to the atmosphere. The airflow is monitored by an in-line flow meter and pressure gage. For all experiments discussed below, the heat lamp is run at a constant power setting and constant elevation $(170 \mathrm{~mm})$ above the top surface of the test articles. The temperature on the heated and unheated sides of the panel is captured with type-K thermocouples attached to an electronic ice point.

At the start of the experiment, the panel is at $24^{\circ} \mathrm{C}$ and the heat lamp is turned on without any active cooling. The temperature on the two surfaces of the panel is recorded at $10 s$ intervals as the temperature rises. Once the temperature on the heated side reaches $110^{\circ} \mathrm{C}$, the coolant flow is activated. The temperature on the panel surfaces is continually monitored until thermal equilibrium is achieved. This experiment is conducted on all five panel densities and for a variety of flow rates.

\section{B. Results and Discussion}

Figure 21 displays data recovered from one of these experiments, which serves as a good example of the type of results observed in each of the experiments. This particular experiment involved a panel with $\rho_{r}=8 \%$ and $\Delta P=20.7 k P a$. The temperature in the uncooled panel rises equivalently on both the heated and the unheated panel. Once the cooling is initiated, the temperature on each side of the panel drops dramatically and quickly establishes a new thermal equilibrium.

Of particular interest for actively cooled structural panels, in general, is the role of the metal foam core in the heat transfer capabilities of the structural thermal protection system. To illustrate this, compare the response displayed in Figure 21 to that shown in Figure 22 for an identical panel that has no foam core at all, only an air gap.

Qualitatively, the responses are very different. In the uncooled foamed panel, the temperature on both sides of the panel rises almost identically, at an average rate of approximately $0.375 \frac{{ }^{\circ} \mathrm{C}}{\mathrm{s}}$. In the uncooled hollow panel, the temperature on the heated side rises nearly twice as fast as that in the un-foamed panel $\left(0.72 \frac{{ }^{\circ} \mathrm{C}}{\mathrm{s}}\right)$, while the temperature on the unheated side rises relatively slowly and moderately.

In the context of hot, uncooled panels, the foamed panel actually provides a disadvantage in performance by assisting in the conduction of heat into the unheated side of the panel, which would be the interior of the vehicle. However, once cooled, the foamed panel provides distinct advantages seen in these plots. First, the foam assists in conducting heat from the heated surface of the MFSP into the cross-section of the coolant flow. Second, with respect to the fact that MFSP's are being considered for a multifunctional solution to the problem of bearing airframe mechanical loads and thermal loads, the panel without a foam core is not a structurally viable component. 
For the thermostructural experiments on actively cooled inconel MFSP's, it is necessary to identify the trends in the thermal performance of MFSP's due to foam density and coolant flow rates. Figure 23 shows the plateau temperatures, the temperatures associated with thermal equilibrium in the heated and actively cooled MFSP's, on the heated and unheated sides of the panels for all densities tested. It is clear from this plot that, under the conditions of the present experiments, the density of the foam does not have an appreciable or clearly defined effect on the thermal performance of MFSP's. So as not to waste effort tailoring thermal performance via foam density, it is valuable to identify that foam density is not a major consideration in the thermal performance of MFSP's and may, therefore, be a variable solely defined by mechanical performance requirements.

Differing pressure drops are required to establish identical flow rates in panels of different foam densities. Thus, instead of examining the effect of flow rate on the thermal performance of MFSP's, the effect of pressure drop is investigated. Figure 24 shows the effect of pressure drop on the plateau temperatures on the heated and unheated sides of an MFSP with $\rho_{f}=8 \%$. Once $\Delta P \geq 30 k P a$, increased pressure drop has only marginal effect on decreasing the panel temperatures. Thus, any pressure drop greater than this value should be appropriate in the thermomechanical experiments. It must be noted from Figure 24 that room temperature $\left(24^{\circ} \mathrm{C}\right)$ can be established on the unheated side of the panel if $\Delta P=70 \mathrm{kPa}$. 


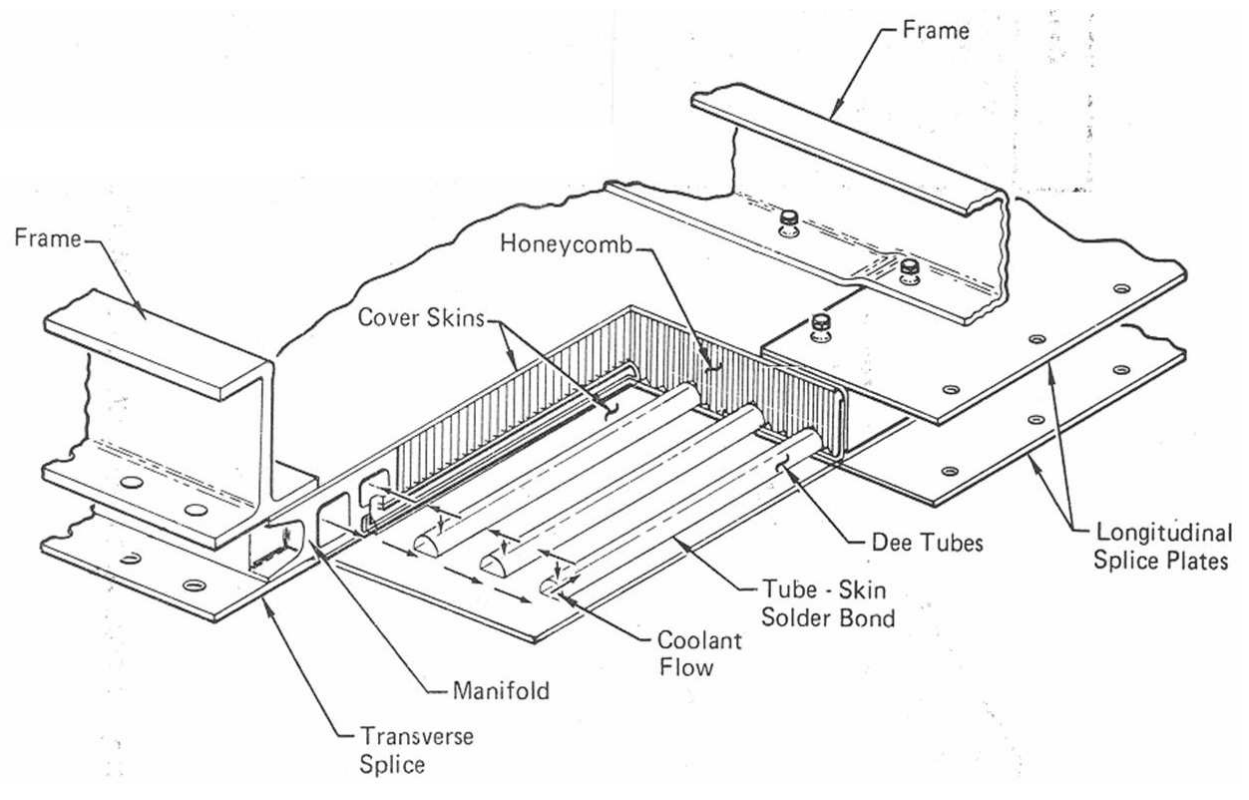

Figure 1. A typical first-generation actively cooled panel concept. ${ }^{3}$

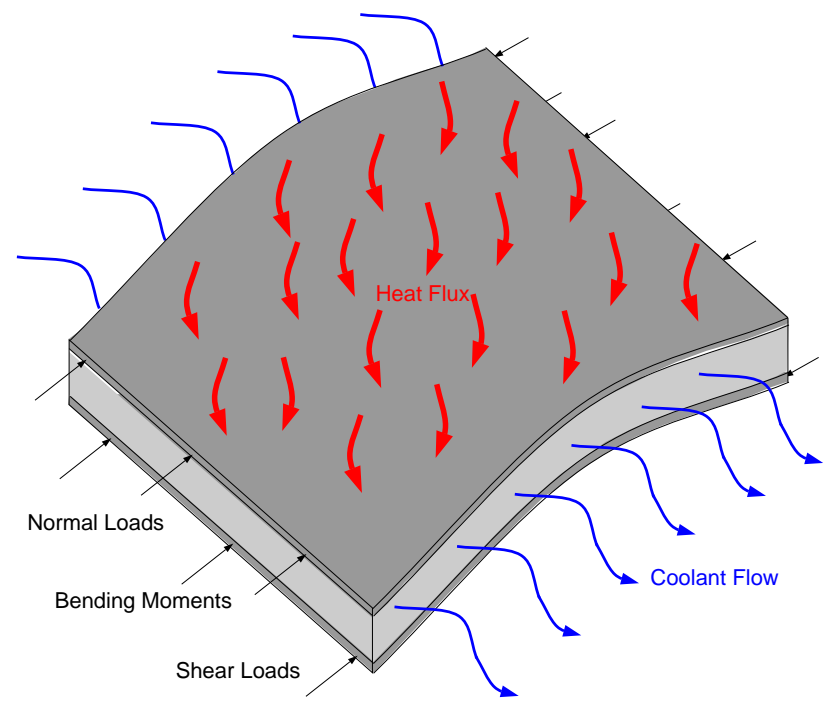

Figure 2. A schematic of an MFSP as it is actively cooled and subjected to airframe flight loads. 


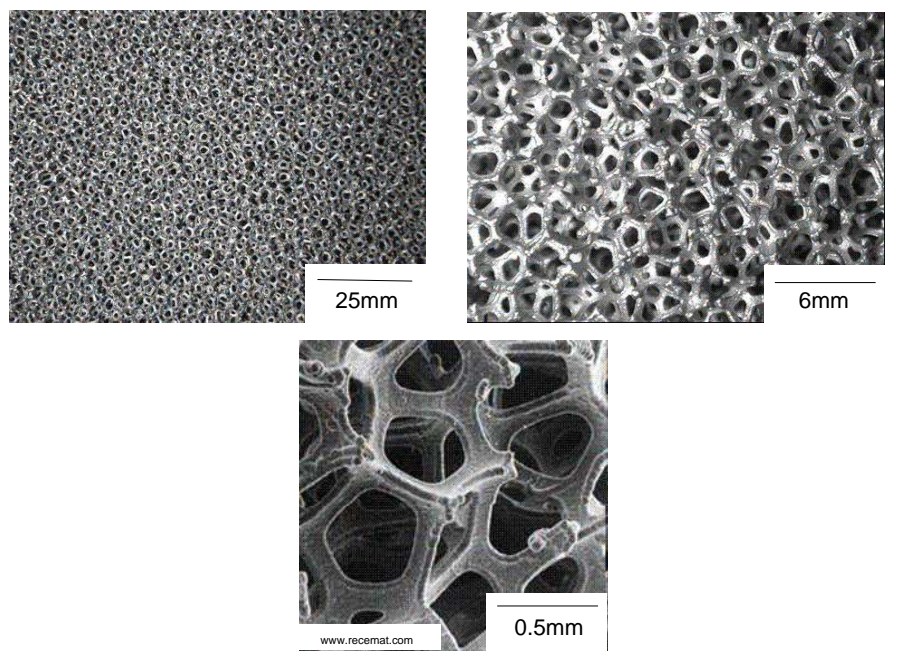

Figure 3. Open cell metal foams.

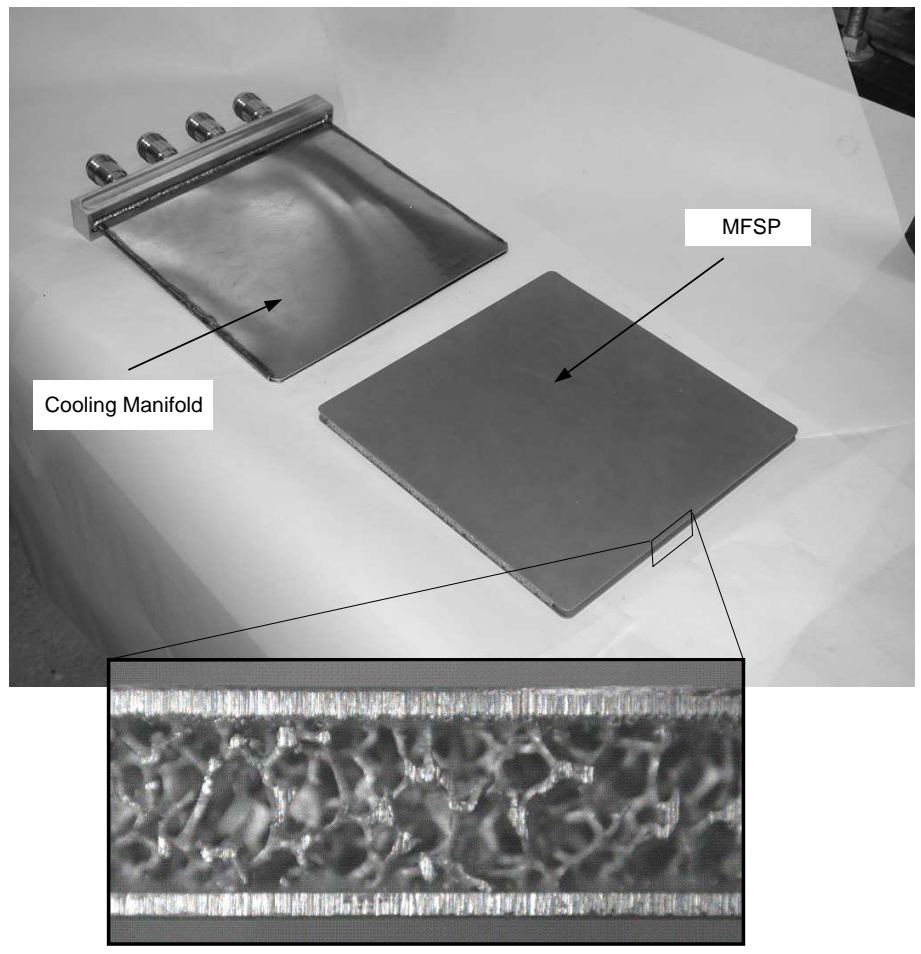

Figure 4. The experimental cooling manifold and MFSP. 


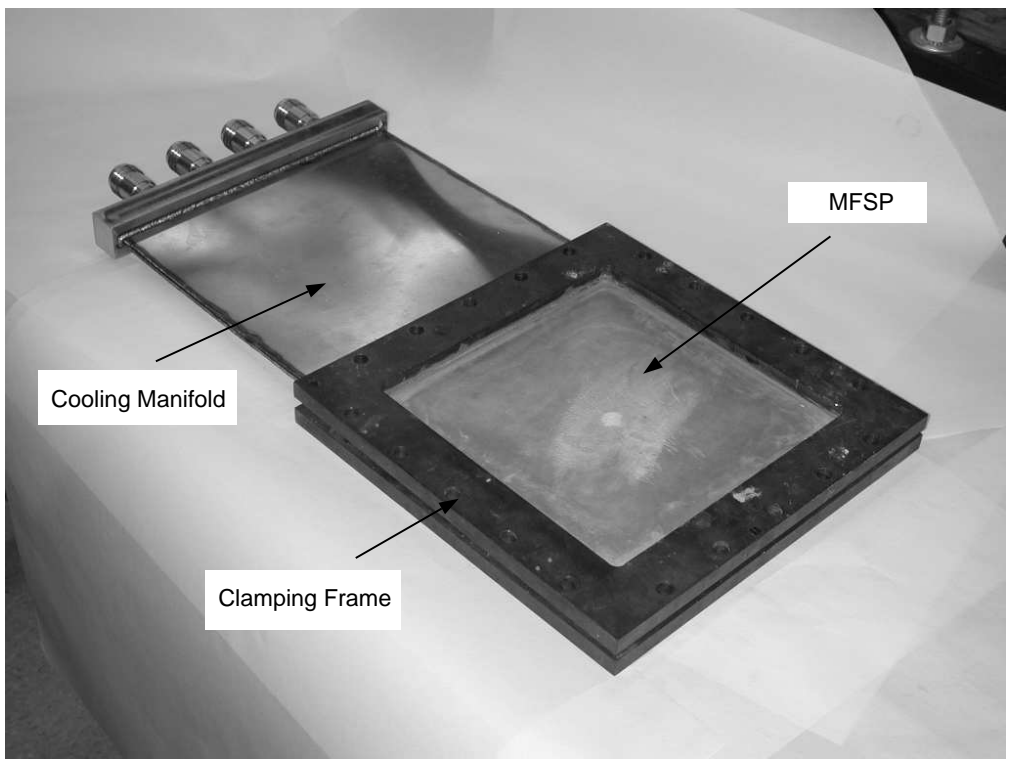

Figure 5. MFSP in the clamping frame with the cooling manifold attached.

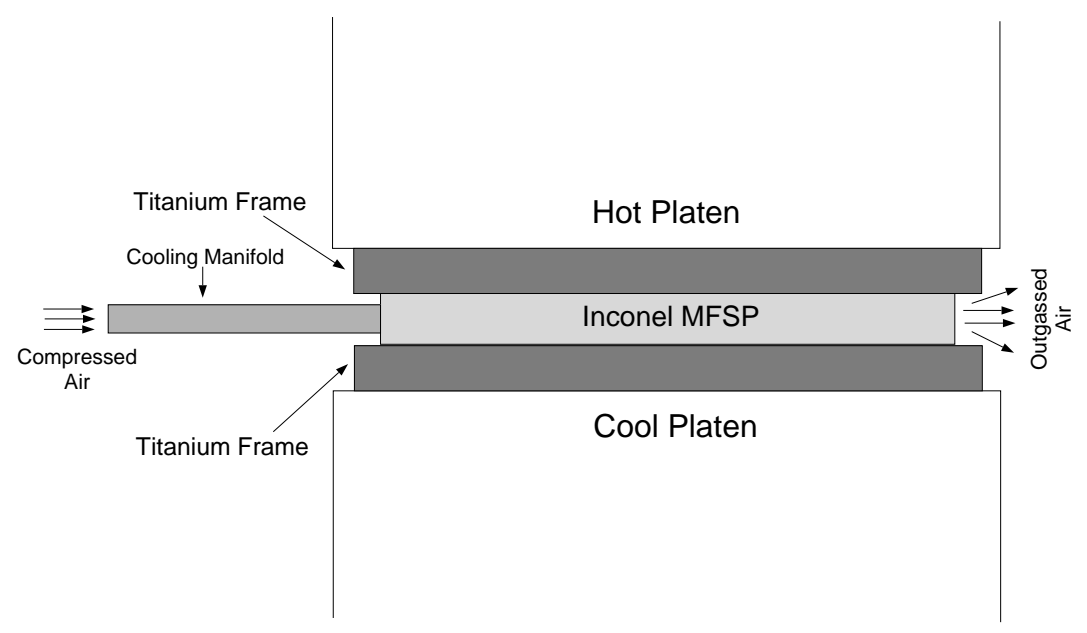

Figure 6. A schematic of the experimental setup for thermal loading of MFSP's with through-the-thickness thermal gradients and active cooling. 


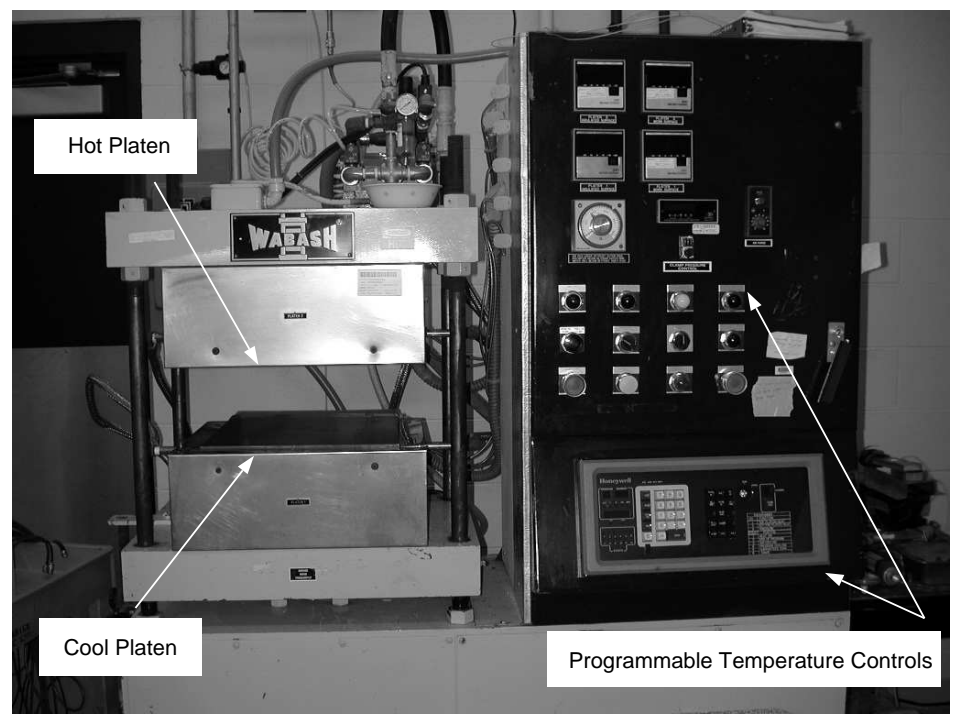

Figure 7. The heat press utilized in the actively cooled experiments.

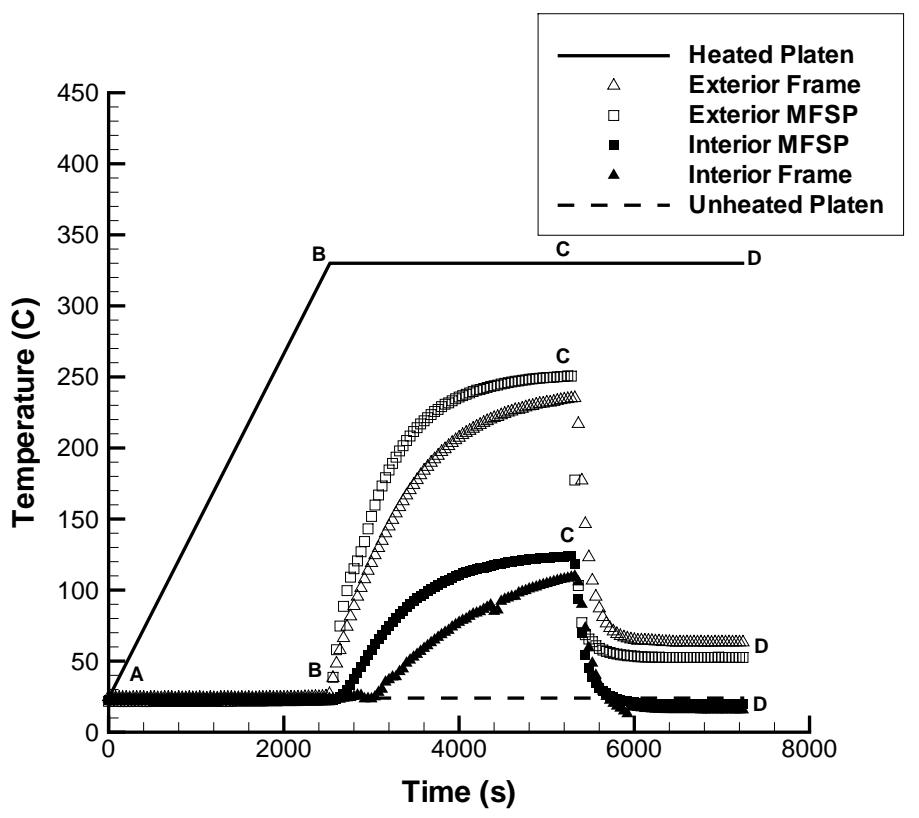

Figure 8. Sample experimental temperature history of the heat press platens, the load frame, and the MFSP. 


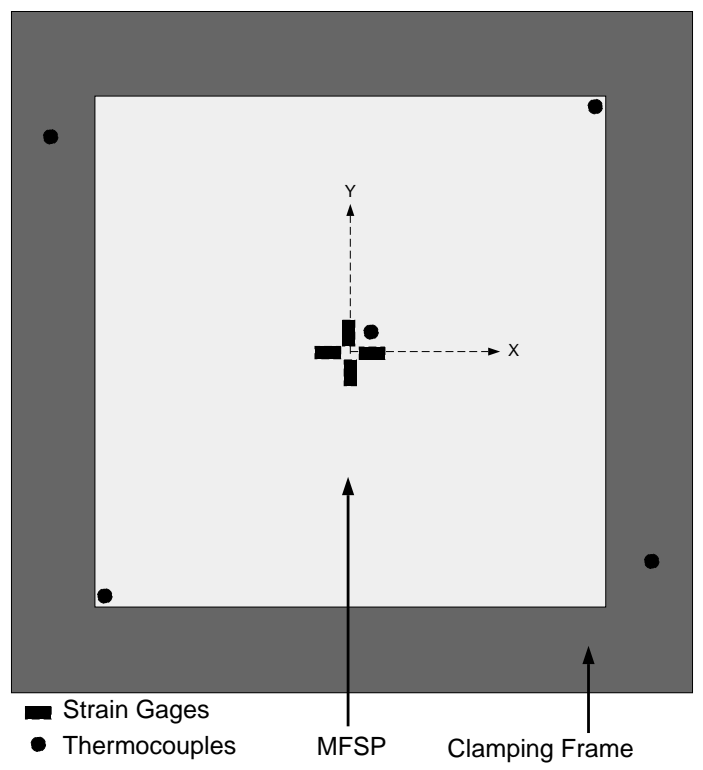

Figure 9. A schematic of the locations of the strain gages and thermocouples employed in the experiments. The locations depicted are utilized on both sides of the panel-frame assembly.

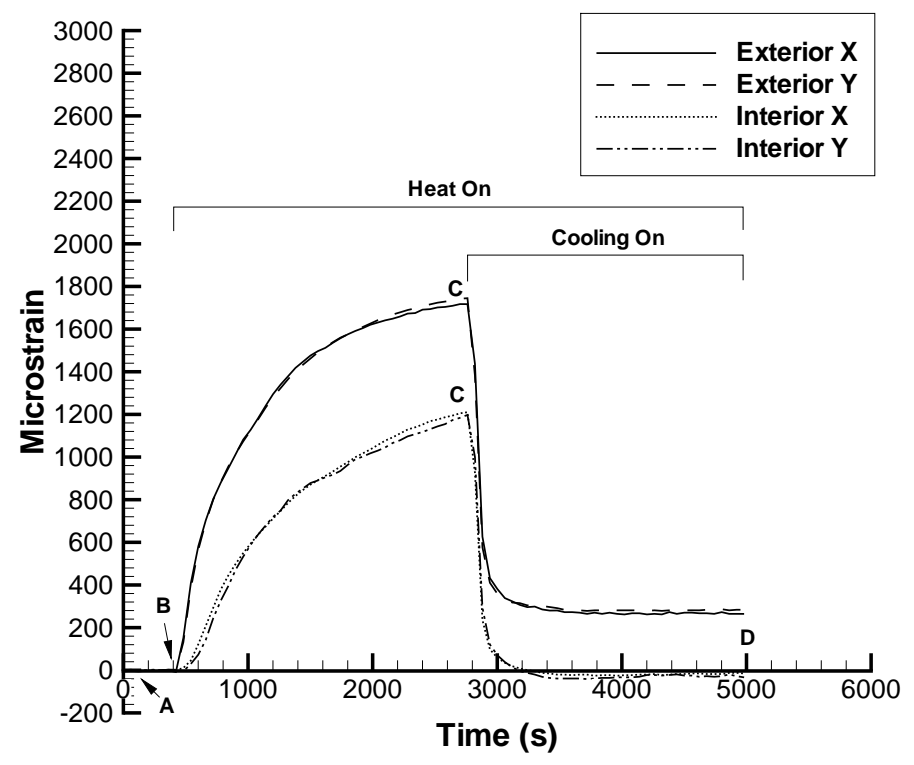

Figure 10. Response of the MFSP, before and during active cooling, under a through-the-thickness thermal gradient with a maximum temperature of $260^{\circ} \mathrm{C}$. 


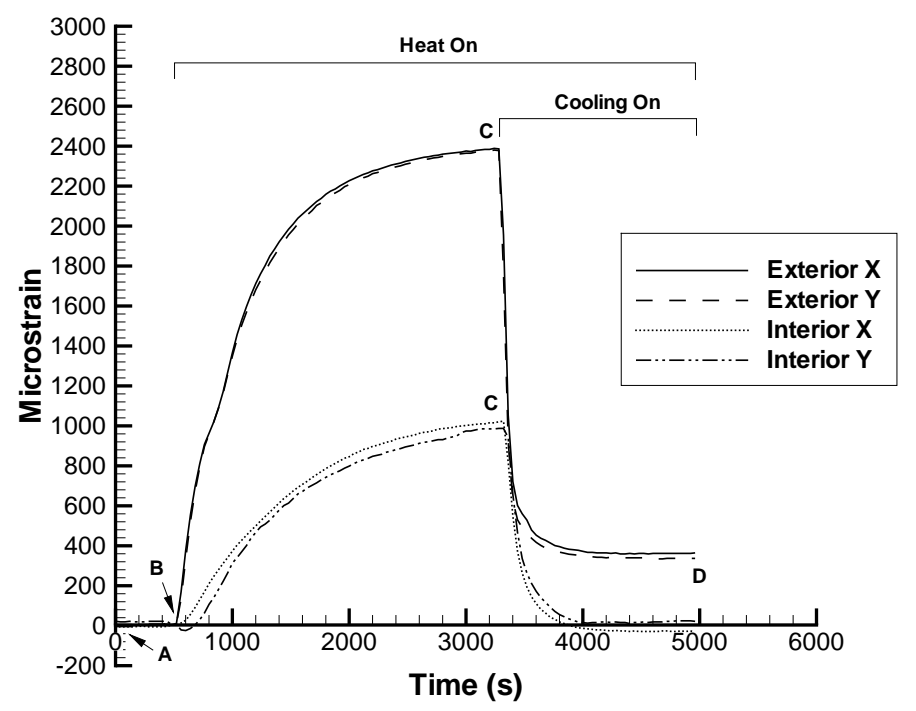

Figure 11. Response of the MFSP, before and during active cooling, under a through-the-thickness thermal gradient with a maximum temperature of $330^{\circ} \mathrm{C}$.

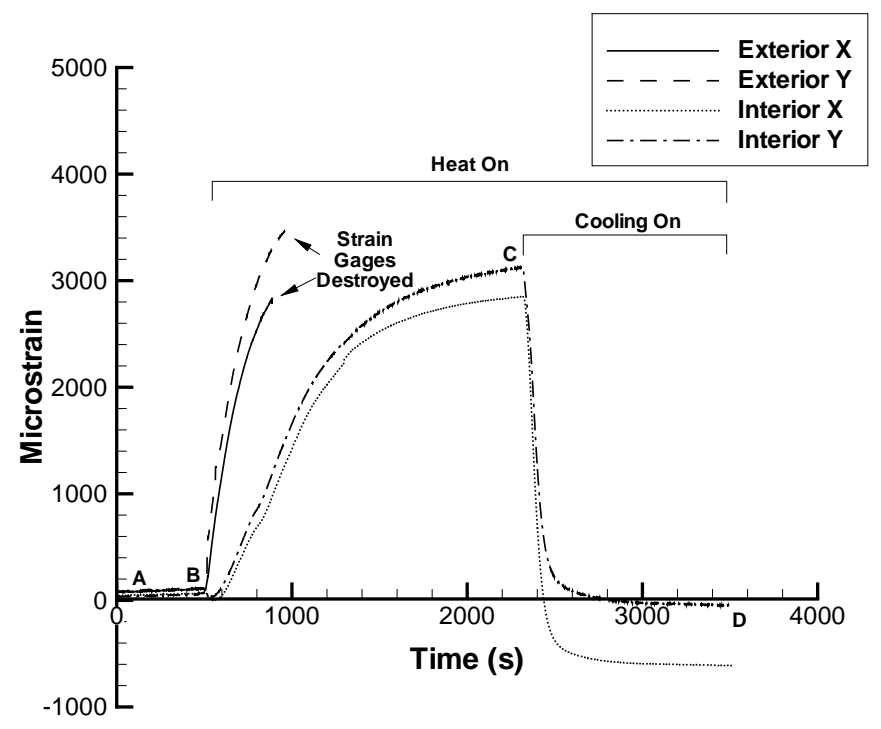

Figure 12. Response of the MFSP, before and during active cooling, under a through-the-thickness thermal gradient with a maximum temperature of $540^{\circ} \mathrm{C}$. 


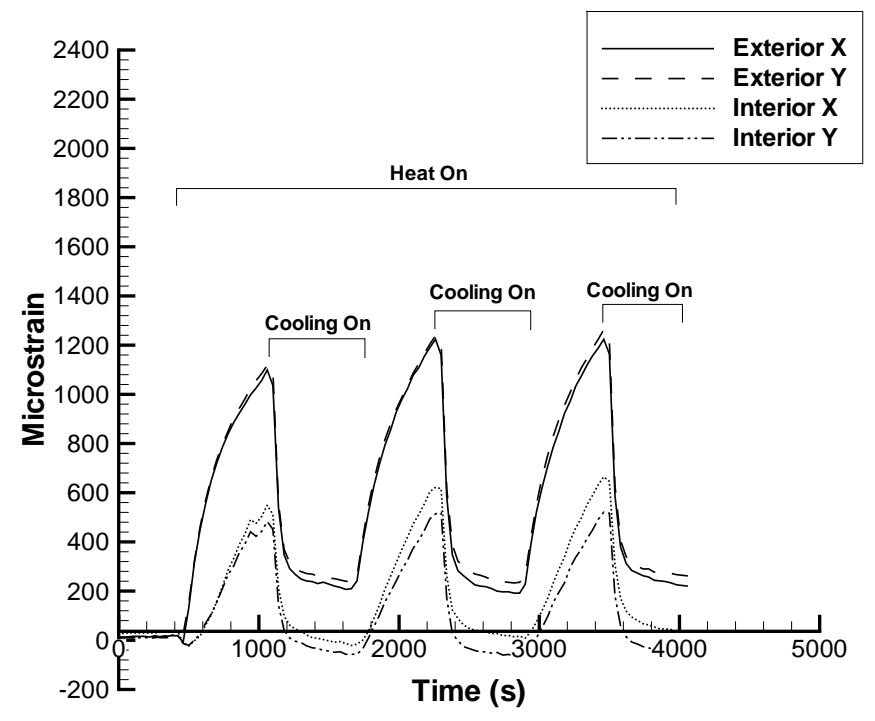

Figure 13. Demonstration of the control of structural deformation under a through-the-thickness thermal gradient with a maximum temperature of $260^{\circ} \mathrm{C}$.

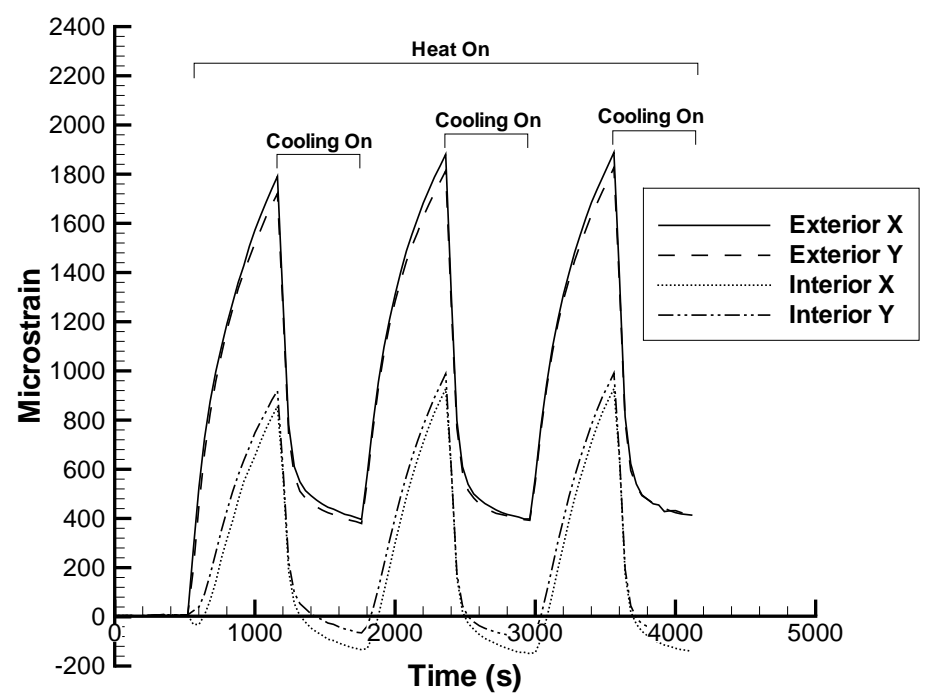

Figure 14. Demonstration of the control of structural deformation under a through-the-thickness thermal gradient with a maximum temperature of $330^{\circ} \mathrm{C}$. 


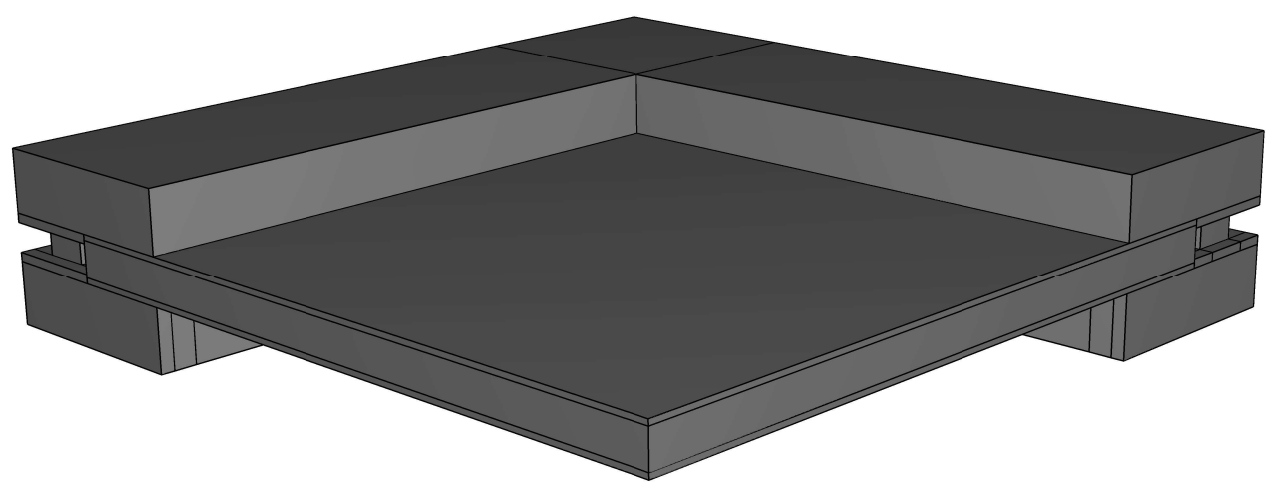

Figure 15. The geometry of the finite element model.

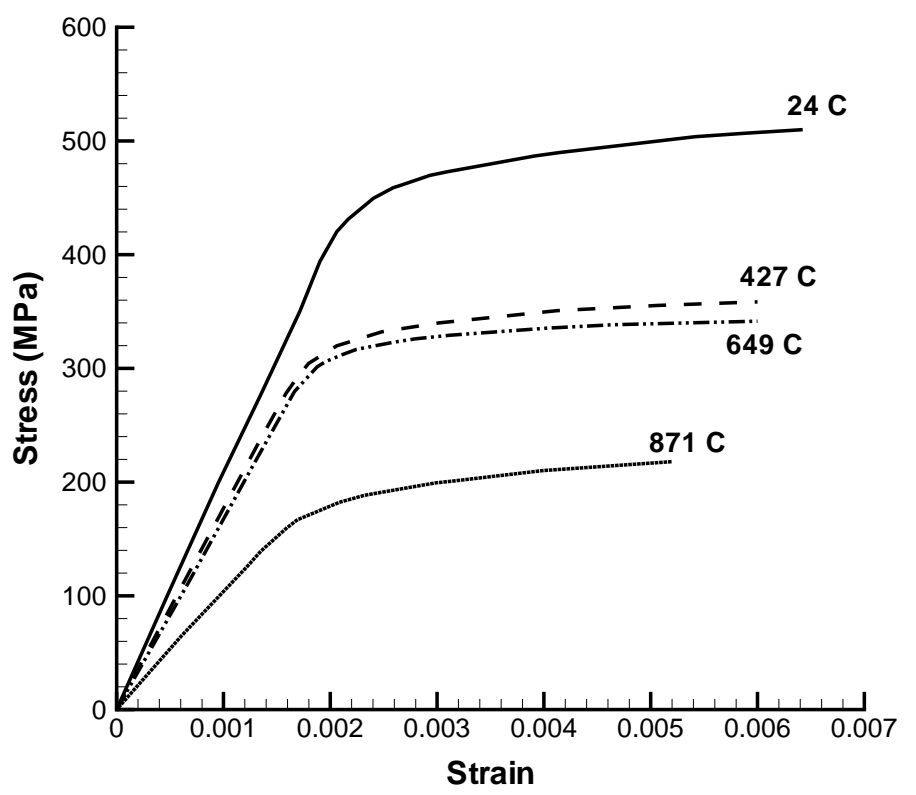

Figure 16. Experimentally determined temperature-dependent stress-strain curves for inconel $625 .{ }^{10}$ 


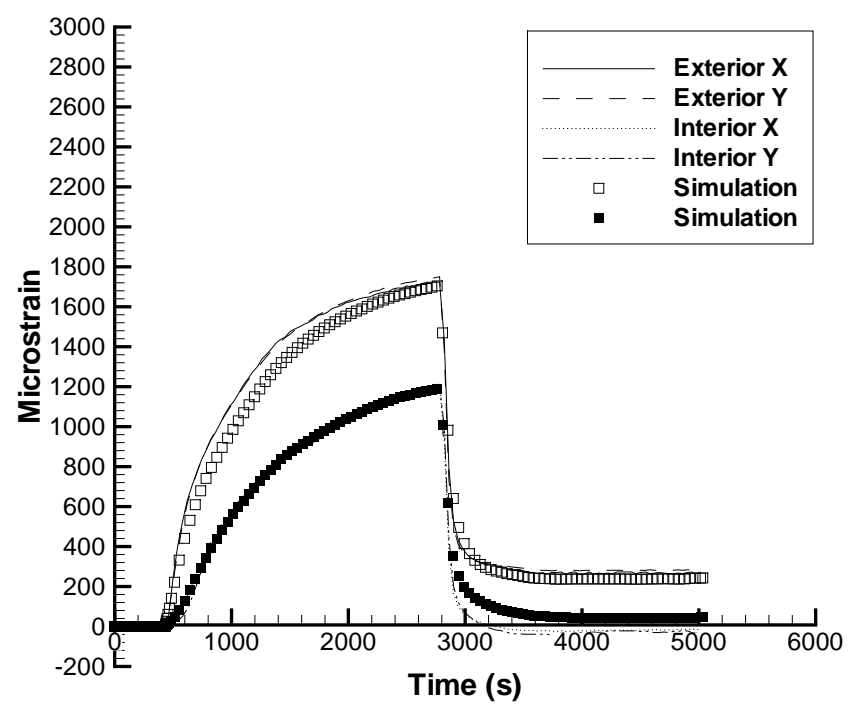

Figure 17. Response of the MFSP, before and during active cooling, under a through-the-thickness thermal gradient with a maximum temperature of $260^{\circ} \mathrm{C}$.

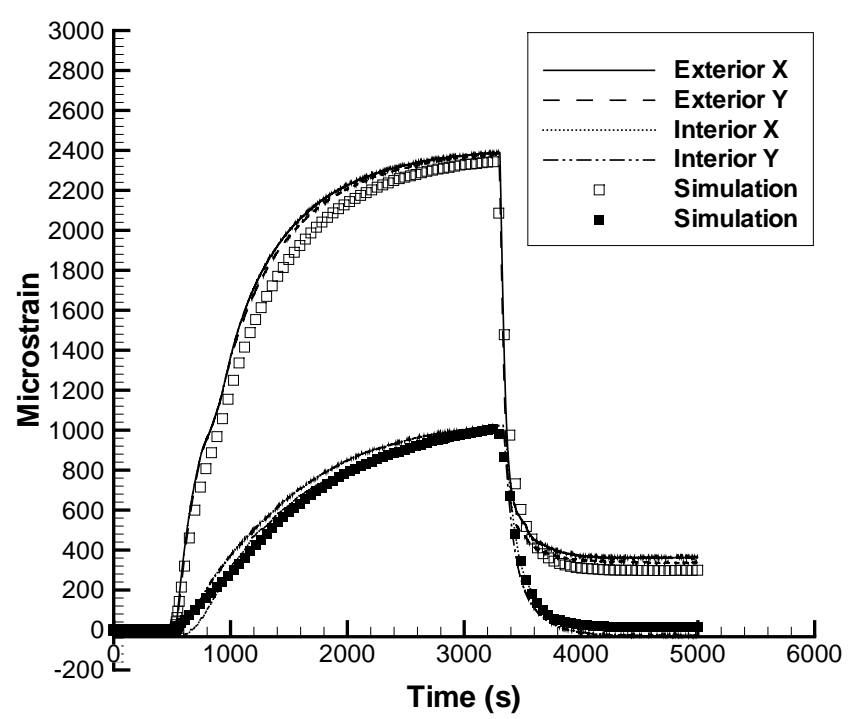

Figure 18. Response of the MFSP, before and during active cooling, under a through-the-thickness thermal gradient with a maximum temperature of $330^{\circ} \mathrm{C}$. 


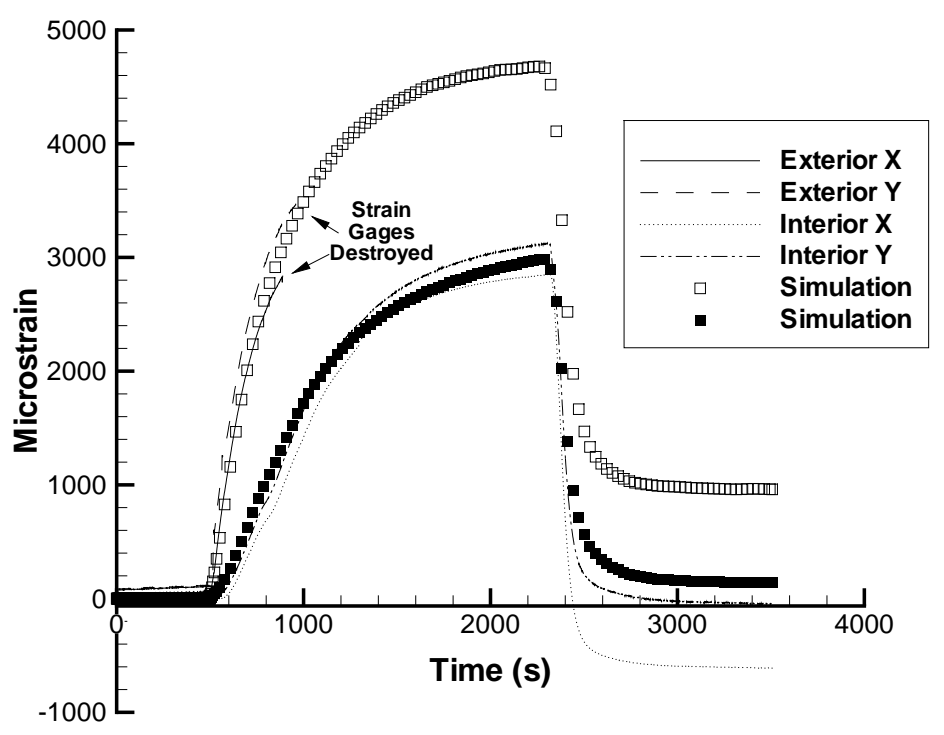

Figure 19. Response of the MFSP, before and during active cooling, under a through-the-thickness thermal gradient with a maximum temperature of $540^{\circ} \mathrm{C}$.

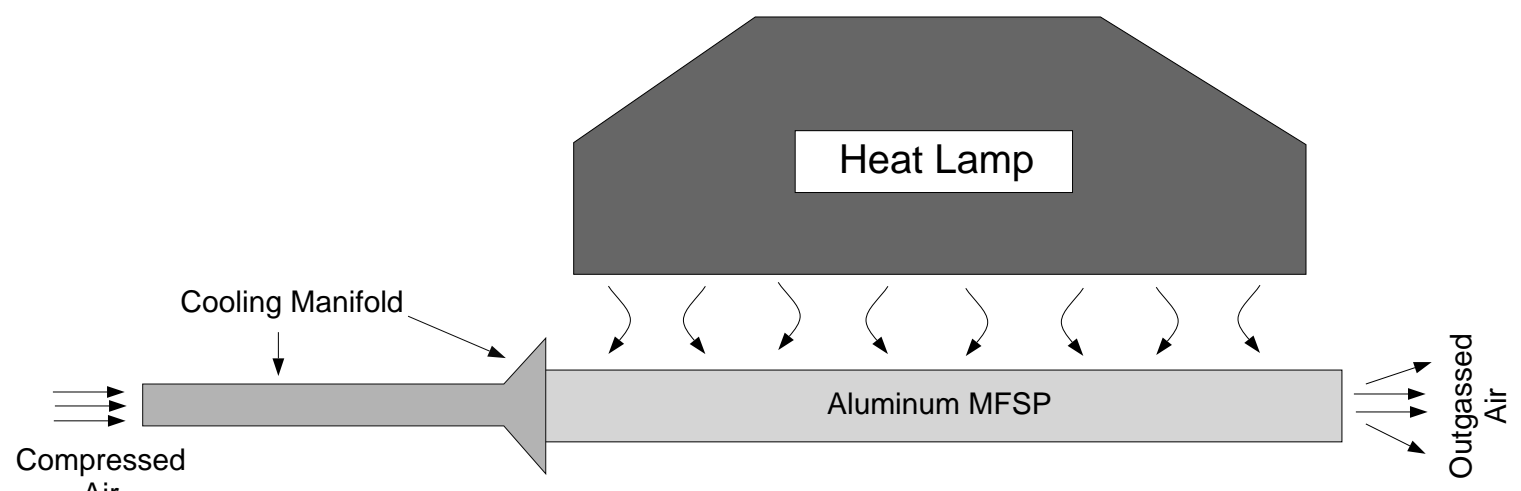

Figure 20. Set up for the parametric study on the thermal performance of MFSP's. 


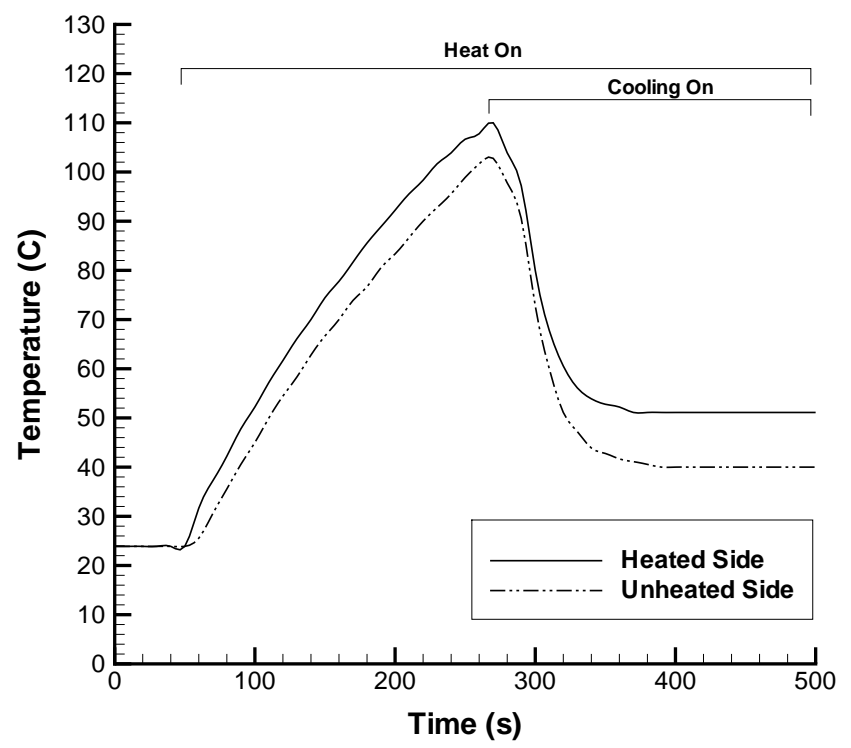

Figure 21. Sample plot of the thermal response of an actively cooled MFSP. Here, $\rho_{r}=8 \%, \Delta P=21 k P a$, $R_{f}=12 S C F M$.

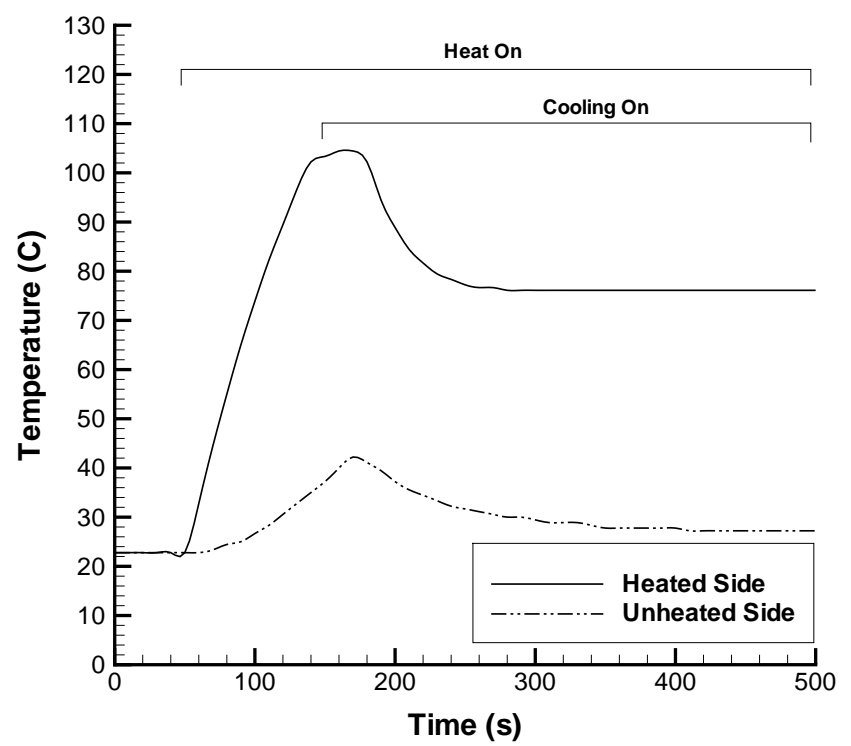

Figure 22. The effect of removing the foam core on the thermal response of the panel. 


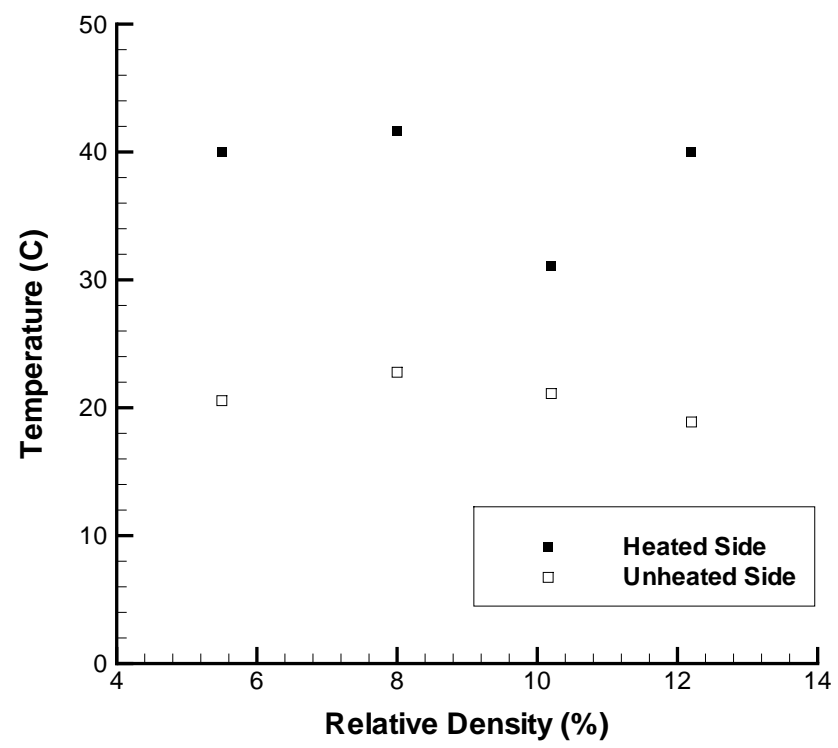

Figure 23. The effect of foam density, $\rho_{r}$, on the thermal equilibrium of the heated MFSP.

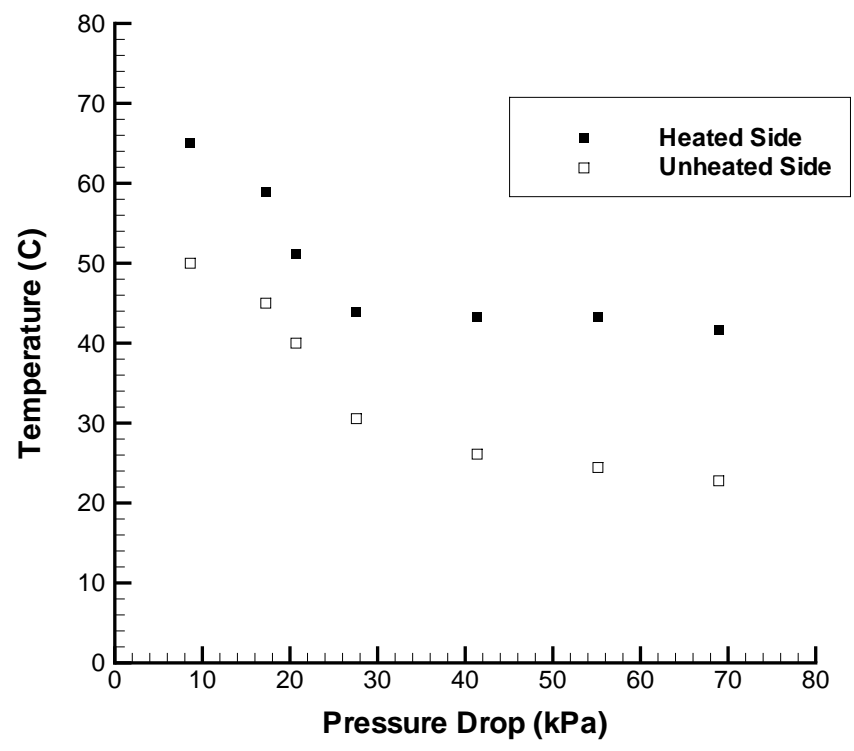

Figure 24. The effect of pressure drop (and flow rate) on the thermal equilibrium of the heated MFSP with $\rho_{r}=8 \%$. 